\title{
Reduced deformability of parasitized red blood cells as a biomarker for anti-malarial drug efficacy
}

\author{
Xiaoyan Deng ${ }^{1}$, Simon P. Duffy ${ }^{1}$, Marie-Eve Myrand-Lapierre ${ }^{1}$, Kerryn Matthews $^{1}$, Aline Teresa Santoso ${ }^{1}$, \\ Yi-Ling Dư ${ }^{2}$ Katherine S. Ryan ${ }^{2}$ and Hongshen $\mathrm{Ma}^{1,3,4^{*}}$
}

\begin{abstract}
Background: Malaria remains a challenging and fatal infectious disease in developing nations and the urgency for the development of new drugs is even greater due to the rapid spread of anti-malarial drug resistance. While numerous parasite genetic, protein and metabolite biomarkers have been proposed for testing emerging anti-malarial compounds, they do not universally correspond with drug efficacy. The biophysical character of parasitized cells is a compelling alternative to these conventional biomarkers because parasitized erythrocytes become specifically rigidified and this effect is potentiated by anti-malarial compounds, such as chloroquine and artesunate. This biophysical biomarker is particularly relevant because of the mechanistic link between cell deformability and enhanced splenic clearance of parasitized erythrocytes.
\end{abstract}

Methods: Recently a microfluidic mechanism, called the multiplexed fluidic plunger that provides sensitive and rapid measurement of single red blood cell deformability was developed. Here it was systematically used to evaluate the deformability changes of late-stage trophozoite-infected red blood cells (iRBCs) after treatment with established clinical and pre-clinical anti-malarial compounds.

Results: It was found that rapid and specific iRBC rigidification was a universal outcome of all but one of these drug treatments. The greatest change in iRBC rigidity was observed for (+)-SJ733 and NITD246 spiroindolone compounds, which target the Plasmodium falciparum cation-transporting ATPase ATP4. As a proof-of-principle, compounds of the bisindole alkaloid class were screened, where cladoniamide A was identified based on rigidification of iRBCs and was found to have previously unreported anti-malarial activity with an $\mathrm{IC}_{50}$ lower than chloroquine.

Conclusion: These results demonstrate that rigidification of iRBCs may be used as a biomarker for anti-malarial drug efficacy, as well as for new drug screening. The novel anti-malarial properties of cladoniamide A were revealed in a proof-of-principle drug screen.

Keywords: Cell deformability, Malaria, Plasmodium falciparum, Drug screening, Biomarkers

\section{Background}

Malaria remains one of the greatest threats to human health. In 2013, there were an estimated 198 million cases resulting in 584,000 deaths [1], of which $90 \%$ occurred in children under the age of five [2]. A number

\footnotetext{
*Correspondence: hongma@mech.ubc.ca

${ }^{1}$ Department of Mechanical Engineering, University of British Columbia, 2054-6250 Applied Science Lane, Vancouver, BC V6T 1Z4, Canada Full list of author information is available at the end of the article
}

of effective anti-malarial drugs and derivatives have been developed. However, the emergence of drug-resistance [3] has prompted the urgent development of new antimalarial compounds as well as protocols to screen these compounds. Existing methods to screen for anti-malarial activity are almost entirely based on parasite survival during the intra-erythrocytic stage and is typically assessed by microscopy, ELISA-based parasite antigen detection, fluorescent DNA-binding dyes, flow cytometry, and PCR of parasite-specific genes [4]. However, the 
measurement of parasite survival provides limited insight into the effectiveness of the candidate drug in vivo, therefore, requiring a large sub-set of compounds to be subsequently evaluated using Plasmodium berghei in murine infection models, using a malarial species different from those that infect humans. Subsequent human clinical trials are costly and are complicated by issues such as toxicity, bioavailability, patient physiology, and side-effects, resulting in extremely low success rates, even for compounds that showed high efficacy in vitro [5]. Owing to the poor success of existing screening methods, there is a need for in vitro screens that align with a common mode of action for anti-malarial compounds in vivo. This type of in vitro method would significantly reduce the burden of animal and human drug trials.

Specific reduced deformability of Plasmodium falciparum parasitized red blood cells (iRBCs) has been well documented. This biophysical change has been attributed to oxidative stress induced by the parasites' metabolism of haemoglobin [6], remodelling of the iRBC cytoskeleton by parasite-derived proteins [7], as well as the presence of the enlarged digestive vacuole in the late stages of infection $[8,9]$. Interestingly, this rigidification of iRBCs enhances their accumulation in micro-anatomical zones and the microcirculation of the spleen, and contributes to their splenic elimination [10]. Furthermore, anti-malarial drugs are likely to exaggerate iRBCs' rigidification and subsequent splenic clearance, by preventing haemoglobin detoxification or increasing intracellular oxidation [11, 12]. Together, these observations suggest that enhanced splenic clearance of iRBCs due to their rigidification might contribute to the mode of action of anti-malarial compounds and would therefore be an important, clinically relevant biomarker for drug efficacy.

Previous studies have reported infection-induced rigidification of iRBCs following exposure to chloroquine or artesunate $[13,14]$. However, parasitized cells may represent a small fraction of the erythrocyte population and this effect may not be detectable by conventional inference of average RBC deformability, based on the viscous properties of blood [15]. The microfluidic multiplexed Fluidic Plunger (MFP) overcomes this limitation by highthroughput measurement of single-cell deformation through microstructures that mimic capillaries in the microvasculature.

The current study aimed to determine whether changes in iRBC deformability correlate with anti-malarial efficacy. It was observed that $\mathrm{iRBC}$ rigidification was a common outcome of the anti-malarial drugs tested. Furthermore, PfATP4 inhibitors that induce rapid clearance of parasitized cells induced the greatest magnitude of iRBC deformability. Finally, in a small screen of bisindole alkaloid compounds, it was shown that changes in iRBC deformability could predict the previously unreported anti-malarial properties of cladoniamide A.

\section{Methods}

\section{Cultivation of Plasmodium falciparum 3D7}

Type A+ blood was collected from healthy donors with written informed consent and approval from the UBC Office of Research Ethics (Network Centre for Applied Development, Canadian Blood Services). Washed erythrocytes were then used to culture $P$. falciparum parasites (strain 3D7) (Network Centre for Applied Development, Canadian Blood Services) at $5 \%$ haematocrit in culture media, consisting of RPMI-1640 medium (Invitrogen, CA, USA) containing 25 mM HEPES (Sigma-Aldrich, St Louis, MO, USA), 0.5 \% AlbuMAX I (w/v) (Invitrogen), $100 \mu \mathrm{M}$ hypoxanthine (Sigma-Aldrich), $12.5 \mu \mathrm{g} / \mathrm{ml}$ gentamicin (Invitrogen) and $1.77 \mathrm{mM}$ sodium bicarbonate (Sigma-Aldrich) at $\mathrm{pH}$ 7.4. Cultures were grown in standard $90 \mathrm{~mm}$ Petri dishes (Corning Life Science, Tewksbury, MA, USA) at a final volume of $10 \mathrm{ml}$ and were kept in a hypoxic incubator (Caron, Marietta, USA) with a standard gas environment of $5 \% \mathrm{CO}_{2}, 1 \% \mathrm{O}_{2}$, and $94 \%$ $\mathrm{N}_{2}$ at $37^{\circ} \mathrm{C}$.

\section{Purification of iRBCs and subsequent drug treatment}

A magnetic purification stand was fabricated based on the design by Kim [16] with some modifications to fit super magnets. LS columns (Miltenyi Biotec, Bergisch Gladbach, Germany) were employed in the positive selection of haemozoin-containing iRBCs (late-stage trophozoite-iRBCs) by loading $30 \mathrm{ml}$ of parasite culture at $2 \%$ haematocrit (v/v) and 5-15\% parasitaemia. Washing media was prepared that was identical to culture media, with the exception of sodium bicarbonate. The columns were washed once with $5 \mathrm{ml}$ washing media and the captured cells were eluted in another $5 \mathrm{ml}$ washing media by centrifugation at $350 \times g$ for 5 min with no brakes. Enrichment purity of late-stage trophozoite-iRBCs was determined Giemsa-staining of an aliquot (Sigma-Aldrich). The remaining cells were suspended in culture media and acclimatized for $30 \mathrm{~min}$ within a hypoxic chamber, prior to incubation with drugs at specified concentrations and incubation times. Anti-malarial compounds were diluted in DMSO to a normalized concentration four times greater than their established EC50 [17-20], as indicated in Table 1 . The $\mathrm{EC}_{50}$ for each candidate anti-malarial drug was not known a priori, so they were each tested at a concentration of $1 \mu \mathrm{M}$. RBCs from healthy donors were evaluated, incubated with $0.01 \%$ DMSO or $\mathrm{ddH}_{2} \mathrm{O}$, as well as each compound to assess whether the observed effect was specific for iRBCs. RBC deformability was determined by multiplexed fluidic plunger and normalized to the control DMSO-treated RBCs. 
Table 1 Individual concentrations and deformability values that were normalized to $\mathrm{DMSO}^{\circ} \mathrm{orddH}_{2} \mathrm{O}$ control for respective anti-malarial drug treatments

\begin{tabular}{|c|c|c|c|c|}
\hline Drug name & $\begin{array}{l}\text { Concentration } \\
\left(\geq 4 \times E C_{50}\right)\end{array}$ & $\begin{array}{l}\text { Median normalized } \\
\text { deformability }\end{array}$ & $\begin{array}{l}\% \text { Median increase } \\
\text { in deformability }\end{array}$ & $P$ value \\
\hline Chloroquine & $1 \mu \mathrm{M}$ & 1.41 & 41 & $<0.0001$ \\
\hline Mefloquine & $1 \mu \mathrm{M}$ & 1.22 & 22 & 0.0002 \\
\hline Pyrimethamine & $20 \mathrm{nM}$ & 1.80 & 80 & $<0.0001$ \\
\hline Proguanil & $100 \mu \mathrm{M}$ & 1.58 & 58 & 0.0052 \\
\hline Artesunate & $10 \mathrm{nM}$ & 1.29 & 29 & 0.0022 \\
\hline Artemether & $8 \mathrm{nM}$ & 1.95 & 95 & $<0.0001$ \\
\hline Dihydroartemisinin & $20 \mathrm{nM}$ & 2.11 & 111 & $<0.0001$ \\
\hline Tetracycline & $100 \mu \mathrm{M}$ & 0.93 & -7 & ns \\
\hline Atovaquone & $250 \mathrm{nM}$ & 1.46 & 46 & 0.0001 \\
\hline (+)-SJ733 & $1.08 \mu \mathrm{M}$ & 5.54 & 454 & $<0.0001$ \\
\hline (-)-SJ733 & $1.08 \mu \mathrm{M}$ & 0.90 & -10 & ns \\
\hline NITD246 & $3.6 \mathrm{nM}$ & 3.34 & 234 & $<0.0001$ \\
\hline
\end{tabular}

\section{Microfluidic measurements of iRBC deformability}

The deformability of iRBCs was measured using the previously developed multiplexed fluidic plunger microfluidic device (Fig. 1a, b) [21]. Briefly, the principle of the fluidic plunger mechanism involves infusing a single cell into a microchannel containing a constriction small enough to significantly deform the cell. When the cell is free flowing in the constriction, pressure applied across the microchannel is distributed along its length. Once the cell reaches the constriction, it forms a temporary seal against the constriction, causing the applied pressure to focus across the cell. Precisely controlling the applied pressure allows an adjustable stress to be applied remotely to a cell and constriction pair. For the experiments described in this study, a microchannel of $2.5 \mu \mathrm{m}$ in width and $3.7 \mu \mathrm{m}$ in thickness was used in order to constrain RBCs in a flat configuration parallel with the microchannel. A constriction aperture of $2.5 \mu \mathrm{m}$ was used to constrain the RBCs and deformed them using pressure applied across the microchannel.

Multiplexing this process involves arranging multiple deformation microchannels in parallel, as well as designing supporting microchannels that ensure that a consistent pressure is applied to all microchannels independent of position and whether they are occupied by a RBC. These supporting microchannels consist of a rectangular detour around parallel deformation, along with longer microchannels leading to the sample inlet and outlet. The rectangular detour structure performs three key functions: (1) pressure applied across the deformation microchannels derives from pressure applied across the inlet and outlet and is reduced by a precise ratio that is set by the relative length of the inlet microchannel and part of the rectangular detour; (2) pressure applied across the deformation microchannels is independent of position in the deformation microchannel array by the symmetric fluidic structure of the rectangular detour; and, (3) pressure applied across the deformation microchannel is made independent of the number of cells being deformed by the relative hydrodynamic resistance of the deformation microchannels and the rectangular detour microchannel. Detailed technical design of the deformation microchannels and the rectangular detour are described in Myrand-Lapierre et al. [21] The device used for this study consists of 34 deformation microchannels in parallel with a rectangular detour microchannel of $25 \mu \mathrm{m}$ in thickness and $250 \mu \mathrm{m}$ in width.

\section{In vitro drug sensitivity test by the SYBR green I assay}

In vitro parasitized erythrocyte cultures were maintained such that the proportion of ring-stage parasites never exceeded $70 \%$ and a parasitaemia of $5-8 \%$ was established prior to drug sensitivity assay. The cells were collected by centrifugation at $350 \times g$ for $5 \mathrm{~min}$ and resuspended in RPMI culture media to a parasitaemia of $0.5 \%$, and the haematocrit was adjusted to $2 \%$ with fresh unparasitized RBCs. Parasitized RBCs were transferred to a 96-well culture plate (Corning, NY, USA) at $200 \mu \mathrm{l} /$ well of $0.5 \%$ parasitized RBCs, at $2 \%$ haematocrit. Each compound was added to the specified concentration (Table 1) and incubated in a hypoxic incubator at $37{ }^{\circ} \mathrm{C}$ $\left(5 \% \mathrm{O}_{2}, 5 \% \mathrm{CO}_{2}, 90 \% \mathrm{~N}_{2}\right.$ ) for $24 \mathrm{~h}$. Uninfected RBCs treated with $0.01 \%$ DMSO (Sigma-Aldrich) and each test compound while iRBCs were also treated with $0.01 \%$ DMSO alone, as controls. Following incubation, the plates were frozen and stored at $-80{ }^{\circ} \mathrm{C}$. Prior to assay, samples were thawed for $1 \mathrm{~h}$ at room temperature and mixed by pipetting. A total of $100 \mu \mathrm{l}$ of each culture was 


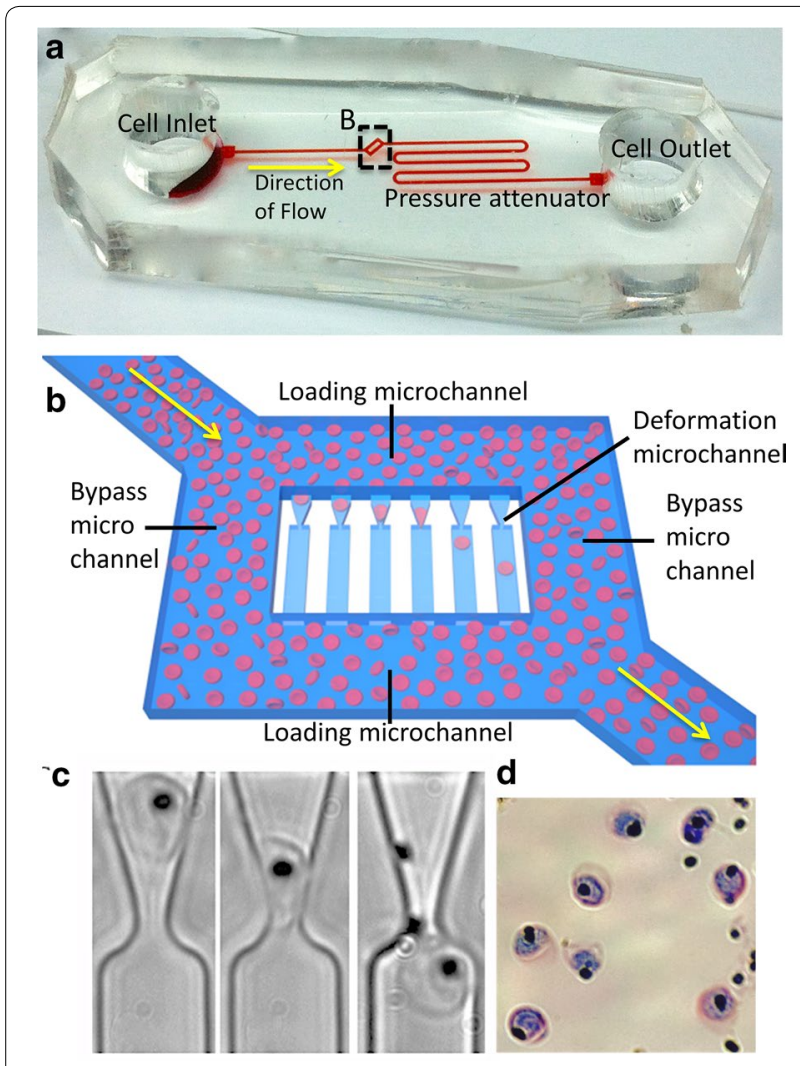

Fig. 1 Overview of the multiplexed fluidic plunger mechanism used to measure trophozoite-infected red blood cells. a The microfluidic device measures $\sim 40 \mathrm{~mm}$ by $20 \mathrm{~mm}$ and consists of a cell inlet and outlet reservoir, a long pressure attenuator and $\mathbf{b}$ deformability microarray, made up of a rectangular microchannel with 2 loading microchannels and 2 bypass microchannels surrounding 34 parallel deformation microchannels with a microconstriction width of $\sim 2.5 \mu \mathrm{m}$ and a height of $\sim 3.7 \mu \mathrm{m}$. RBCs are loaded into the deformation microchannel under very low pressure. The pressure is then incrementally increased until the cell transits the microconstriction. c The pressure required for the trophozoite-infected RBC (RBCs with a black pigment) to transit the microconstriction is indicative of the cell's deformability [21]. d Giemsa-stained blood smear confirming the pigmented iRBCs measured by the device are trophozoites

mixed with $100 \mu \mathrm{l}$ of $1 \times$ SYBR in $2 \times$ Lysis buffer [ $20 \mathrm{mM}$ Tris; $0.008 \%$ Saponin (w/v); $0.08 \%$ Triton X-100 (w/v)] and transferred to a new 96-well plate. Fluorescence signal was determined in duplicate using a Biomek FX Multimode Detector (Beckman Coulter, Pasadena, CA, USA) at an excitation wavelength of $485 \mathrm{~nm}$ and an emission wavelength of $535 \mathrm{~nm}$.

\section{Statistical analysis}

Median RBC deformability and interquartile range (IQR) was calculated using Graphpad Prism v5. A Student $t$ test was used to assess statistical significance between normally distributed $\mathrm{RBC}$ deformability profiles. The relationship between changes in RBC deformability and anti-malarial drug incubation time or concentration was evaluated by linear regression, while non-linear regression analysis was used to analyse the in vitro $\mathrm{IC}_{50}$ of new compounds on parasites.

\section{Results \\ Multiplexed fluidic plunger detected time-dependent rigidification of chloroquine-treated iRBCs}

Alteration in RBC deformability after $P$. falciparum infection and anti-malarial drug treatment was assessed using the MFP device [21], which measures the deformability of single RBCs based on the pressure required to transit a funnel-shaped microconstriction (Fig. 1). To evaluate the sensitivity of this system for measuring drug-induced changes in RBC deformability, the deformability of purified $P$. falciparum 3D7 iRBCs was determined over a range of chloroquine concentrations by measuring the median transit pressure through a microscale funnel (Fig. 2a). While chloroquine did not affect the median transit pressure for uninfected RBCs (Additional file 1: Figure S1), iRBCs required significantly greater transit pressure at $1 \mu \mathrm{M}(\mathrm{p}=0.0002)$ and $1.5 \mu \mathrm{M}(\mathrm{p}=0.0006)$ of chloroquine treatment, compared to untreated controls. Both untreated and chloroquine-treated iRBCs showed a time-dependent rigidification. However, following $4 \mathrm{~h}$ of incubation, the chloroquine-treated iRBCs were significantly more rigid than untreated iRBCs (Fig. 2b, $\mathrm{p}=0.0026$ ). Furthermore, there is a direct linear correlation between chloroquine incubation time and iRBC transit pressure (Fig. 2c, $\mathrm{R}^{2}=0.9784$ ).

\section{Measurement of iRBC deformability following treatment with a panel of conventional anti-malarial compounds}

Previous studies have reported that both chloroquine and artesunate treatment contribute to a specific rigidification of iRBCs $[13,14]$. However, it was uncertain whether this was a common feature of anti-malarial compounds or whether this phenomenon was restricted to these specific drugs. To assess whether iRBC rigidification was a universal outcome of anti-malarial drug treatment, the effect of 12 established anti-malarial compounds on iRBC deformability were systematically assessed. Purified iRBCs were incubated with drugs at a specific concentration $\left(\geq 4 \times \mathrm{EC}_{50}\right)$ for $4 \mathrm{~h}$ and alteration in the biophysical characteristics of the cell were assessed by MFP. None of these anti-malarial compounds significantly altered the deformability of uninfected RBCs (Additional file 2: Figure S2). Suspensions of iRBCs in $0.001 \%$ DMSO and $\mathrm{ddH}_{2} \mathrm{O}$ were used as baseline controls for this experiment and there was no significant difference in median transit pressure between these control iRBCs. In contrast to untreated controls, a significant increase in median 

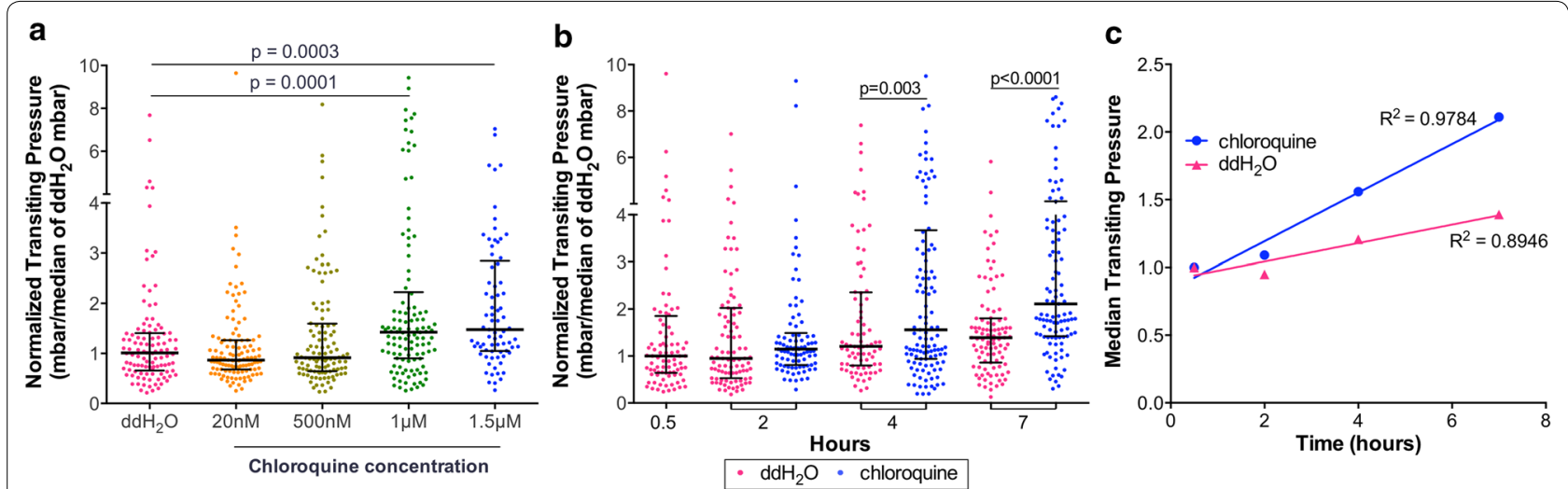

Fig. 2 Chloroquine exposure significantly decreases the deformability of trophozoites-infected red blood cells in a dose- and time-dependent manner. a Purified trophozoites were incubated with a range of chloroquine concentrations for $4 \mathrm{~h}$. Each data point represents the transit pressure for one trophozoite IRBC and a significant increase in transit pressure was observed a 1 and $1.5 \mu$ M. b Purified trophozoites were incubated with $1 \mu \mathrm{M}$ chloroquine for at 2, 4 or $7 \mathrm{~h}$. Treated and untreated iRBCs showed time-dependent loss of deformability, with a significant increase in transit pressure by $4 \mathrm{~h}(\mathrm{p}=0.0026)$, compared to untreated iRBCs. c There is a positive correlation between the median transiting pressure of iRBCs incubated with $\left(R^{2}=0.9784\right)$ and without $\left(R^{2}=0.8946\right)$ chloroquine treatment and time of incubation

transit pressure was observed for all drug-treated iRBCs, except tetracycline and the (-)-SJ733 enantiomer that serves as a negative control with no anti-malarial activity (Fig. 3, p < 0.001).

It was observed that the different anti-malarial compounds had different effects on the deformability of iRBCs (Table 1). For example, while mefloquine and chloroquine are related compounds, mefloquine only reduced $\mathrm{iRBC}$ deformability by $22 \%$, while chloroquine induced $44 \%$ reduction in iRBC deformability. Similarly, it was observed that each of the artemisinin derivatives induced significant rigidification of iRBCs and among them, dihydroartemisinin resulted in the least deformable iRBCs (111\%). The greatest rigidified iRBCs resulted from treatment with NITD 246 and the active (+)-SJ733 enantiomer, showing 228 and $469 \%$ loss in deformability, respectively, relative to the control (Fig. 3; Table 1).

\section{Screen of bisindole alkaloid compounds for anti-malarial activity}

Collectively, these data suggested that $\mathrm{ARBC}$ rigidification could be an alternative method for anti-malarial drugs screening. Therefore, as a proof-of-principle, a group of bisindole alkaloid compounds were tested, which derive biosynthetically from the oxidative dimerization of tryptophan [22-24] for their effects on iRBCs' rigidification. Specifically, cladoniamide A, xenocladoniamide D, arcyriaflavin A, and K252c were examined and it was observed that (Fig. 4a) cladoniamide A imparted $17 \%$ greater rigidification of iRBCs than did chloroquine (Fig. 4b, $\mathrm{p}<0.0001)$. Xenocladoniamide D and K252c resulted in a slight non-significant rigidification of $\mathrm{i} B C$ relative to the
DMSO control and arcyriaflavin A had no effect. None of these compounds affected the deformability of uRBCs (Additional file 3: Figure S3).

To assess whether the specific induced $\mathrm{IRBC}$ rigidification observed for cladoniamide A correlated with anti-malarial activity, an in vitro SYBR-Green fluorescence assay was performed to measure the effect of each drug on parasitaemia and generated dose response curves to determine the $\mathrm{IC}_{50}$ for each compound. Consistent with the deformability analysis chloroquine $\left(\mathrm{IC}_{50}=28 \mathrm{nM}\right)$ and cladoniamide $\mathrm{A}\left(\mathrm{IC}_{50}=7.8 \mathrm{nM}\right)$ displayed anti-malarial activity at a significantly lower dose than xenocladoniamide $\mathrm{D}\left(\mathrm{IC}_{50}=2.27 \mu \mathrm{M}\right)$ and $\mathrm{K} 252 \mathrm{c}\left(\mathrm{IC}_{50}=1.9 \mu \mathrm{M}\right)($ Fig. $4 \mathrm{c})$. The anti-malarial activity of cladoniamide A has not been previously reported. However, it is interesting to note that cladoniamide A was recently reported to target a V-Type $\mathrm{H}^{+}$-ATPase in yeast [25]. In the Plasmodium parasite the V-type $\mathrm{H}^{+}$ATPase maintains the intracellular $\mathrm{pH}$ and disruption of this transporter contributes to acidification of the parasite [25]. Another prominent anti-malarial drug target is PfATP4 which employs efflux to maintain a low parasite intracellular $\mathrm{Na}^{+}$concentration in $\mathrm{Na}^{+}$-rich blood. However, it has been reported that malaria V-Type $\mathrm{H}^{+}$ATPase activity is $\mathrm{Na}^{+}$-dependent, suggesting mechanistic link between these transporters and PfATP4 [26]. Together, these findings suggest that alterations in iRBC deformability correlate with anti-malarial activity. This rapid deformability-based screen was employed to identify the putative V-Type $\mathrm{H}^{+}$-ATPase inhibitor cladoniamide $\mathrm{A}$ as a compound with potent anti-malarial activity. 


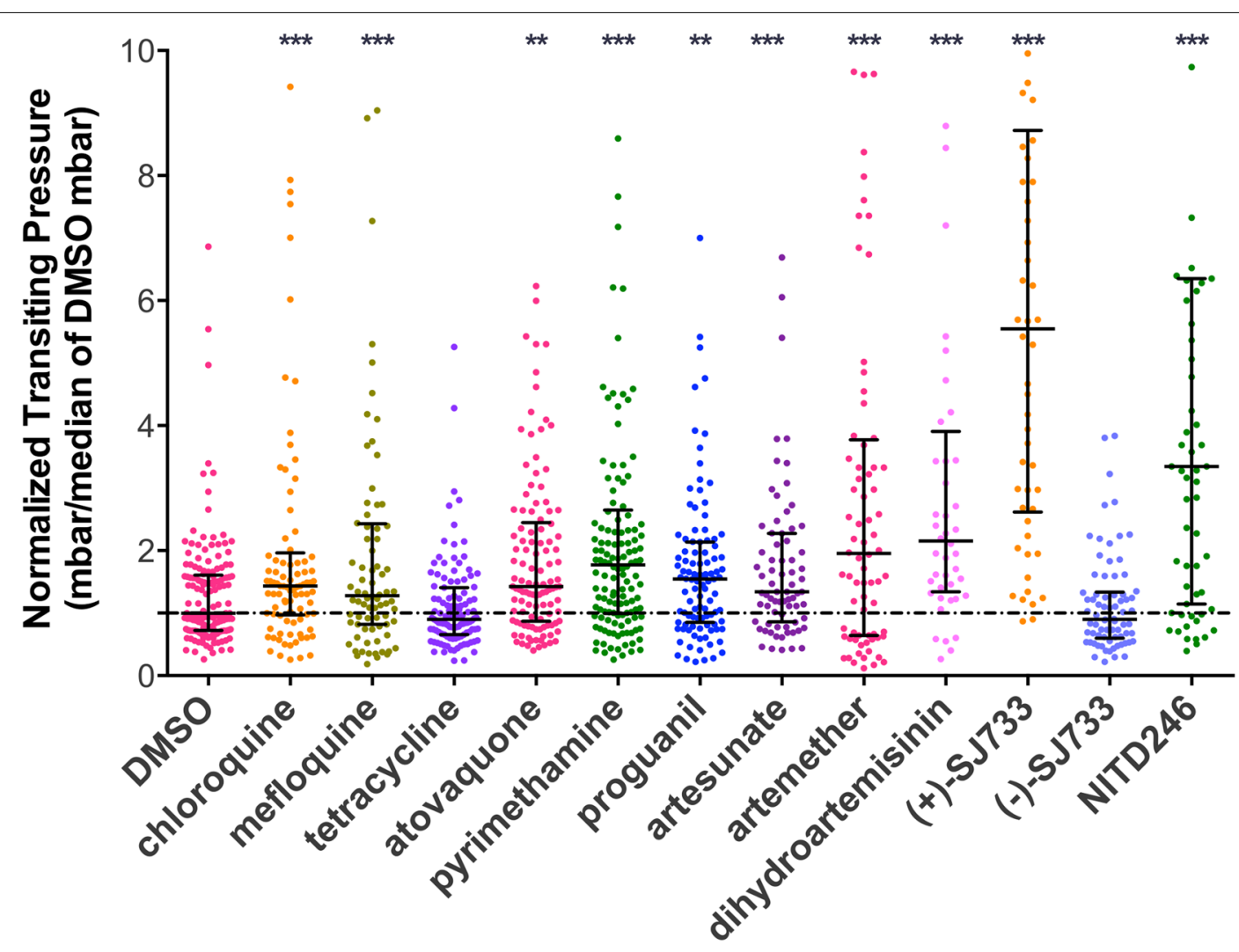

Fig. 3 Anti-malarial drugs impair the deformability of trophozoite-infected red blood cells. The deformability of purified trophozoite-iRBCs was significantly diminished by treatment with all anti-malarial drugs tested, except tetracycline. Treatment with the novel spiroindolone NITD246 and DHIQ-(+) SJ733 showed the highest transiting pressure required for the iRBCs to squeeze through the microconstrictions of the device. ${ }^{* *} p<0.001,{ }^{* * *} p<0.0001$

\section{Discussion}

This study involved systematic assessment of the effect of established clinical and pre-clinical anti-malarial compounds on the deformability of parasitized RBCs. It was observed that decrease in cell deformability was a common outcome of nearly all established anti-malarial compounds tested. Furthermore, using specific reduced iRBC deformability as a biomarker for drug efficacy, cladoniamide A was identified as a candidate novel anti-malarial compound. Further investigation indicated that this compound has an $\mathrm{IC}_{50}$ that is lower than chloroquine.

While most of the anti-malarial compounds examined in this study corresponded with a decrease in iRBC deformability, they likely do so by distinct cellular mechanisms. The Plasmodium parasite metabolizes the haemoglobin of the host erythrocyte and generates toxic haem as a byproduct. Normally, the parasite detoxifies the host cell by biocrystallization of haem into haemozoin, but chloroquine inhibits this process and inhibits haem degradation to collectively contribute to the accumulation of haem within the host cell [27]. Intracellular haem contributes to oxidative stress in the iRBC, which is thought to be directly toxic to the parasite based on the observed sensitivity of $P$. falciparum to pro-oxidants [28], such as nitric oxide [29], hydrogen peroxide and superoxide [30]. Furthermore, oxidative stress contributes to reduced cell deformability by membrane lipid peroxidation [11]. Also, quinoline anti-malarial drugs, such as chloroquine and mefloquine, induce haem association with the cell membrane, which further impairs the integrity of the membrane [28]. The observation that mefloquine has a more subtle impact on iRBC deformability than chloroquine is consistent with observation that mefloquine is a less effective inhibitor of haem degradation [28]. In contrast to the quinolone drugs, the mechanism for artesunate anti-malarial activity is not well established. However, artemisinin derivatives, such as artesunate, arthemether and dihydroartemisinin, are thought to be rapidly hydrolyzed or demethylated to their active metabolite, dihydroartemisinin [31,32], which is therapeutically active against malaria infection [33]. Artemisinins may also target sarco/endoplasmic reticulum $\mathrm{Ca}^{2+}$-ATPase, P. falciparum phosphatidylinositol3-kinase and haemoglobin digestion [34]. These cellular processes stimulate reactive oxygen species (ROS) that 


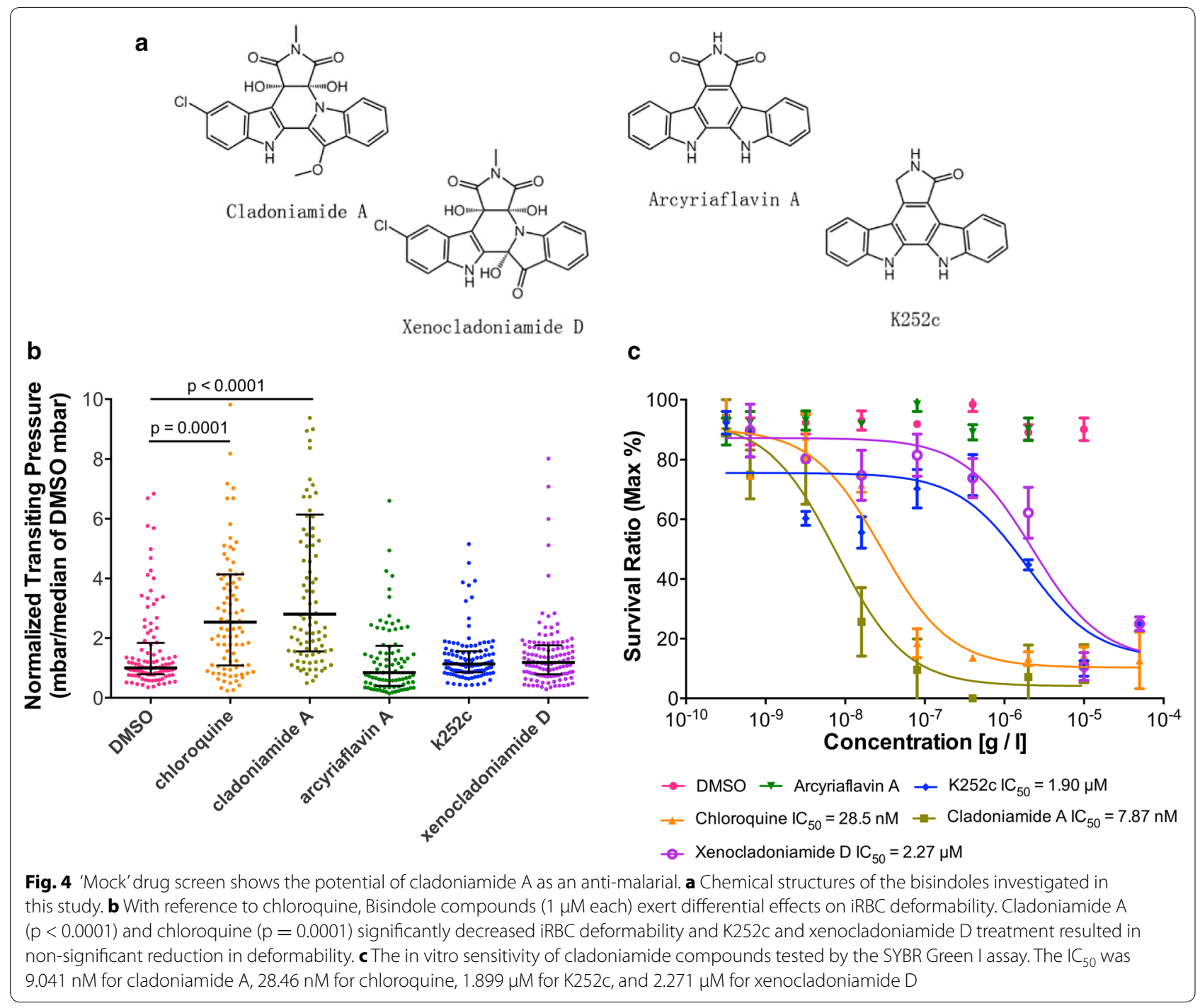

oxidize membrane thiols and contribute to reduced cellular deformability [12].

NITD 246 and the active (+)-SJ733 enantiomer induced the greatest rigidification of iRBCs. Both active molecules belong to the novel anti-malarial spiroindolone family that target PfATP4, a P-type ATPase [20, 35, 36]. The uptake of important nutrition by invading parasites allows $\mathrm{Na}^{+}$influx and $\mathrm{K}^{+}$efflux at the same time [36]. PfATP4, a parasite plasma membrane protein, helps intra-erythrocytic parasites maintain a low cytosolic $\mathrm{Na}^{+}$by extruding $\mathrm{Na}^{+}$against an inward gradient in exchange for $\mathrm{H}^{+}$entry [36]. NITD246 and (+)-SJ733 work as inhibitors of PfATP4 in nanomolar concentrations and perturb efflux of $\mathrm{Na}^{+}$and coupled influx of $\mathrm{H}^{+}$by PfATP4 into the parasites, which increases both intracellular $\mathrm{pH}$ and sodium concentrations, resulting in parasite swelling and death $[20,36]$ as well as a spherical morphology of the RBC [20]. The (-)-SJ733 enantiomer was unable to reduce the deformability of iRBCs, consistent with its low potency against malaria in vitro [20] and highlights the specificity of the anti-malarial property to (+)-SJ733.

As a proof-of-principle, a group of bisindole alkaloid compounds were screened. Specifically, cladoniamide A, xenocladoniamide D, arcyriaflavin A, and $\mathrm{K} 252 \mathrm{c}$ were examined. Cladoniamide A is a nanomolar cytotoxic agent, thought to target vacuolar $\mathrm{H}^{+}$-ATPase [25], xenocladoniamide $\mathrm{D}$ is an inhibitor of the colon cancer cell line HCT-116 [23], arcyriaflavin A inhibits human cytomegalovirus replication in vitro, and K252c inhibits many protein kinases, such as protein kinase $C$ [37]. None of these compounds has been previously reported to have antimalarial properties and none of them affected the deformability of uRBCs. The observation that cladoniamide A induces a dramatic rigidification of iRBCs and that this corresponds with a potent anti-malarial effect serves to support the hypothesis that reduced iRBC deformability is indicative of anti-malarial drug efficacy. 


\section{Conclusion}

These results suggest that specific rigidification of iRBCs is a feature common to the anti-malarial compounds tested. Moreover, the propensity for less deformable cells to be retained within the interendothelial clefts of the spleen [10], suggest that changes in iRBC deformability may contribute to the mode of action of anti-malarial compounds. Support for this assertion can be drawn from studies where chloroquine-mediated changes in RBC deformability promote more rapid clearance of the rigid cells [14]. Furthermore, the observation that the spiroindolones NITD246 and (+)-SJ733 induced the greatest magnitude of iRBC rigidification is consistent with observations that the primary mode of action for these PfATP4 inhibitors is by host-mediated clearance [20]. The progressive rigidification of parasitized RBCs has previously been attributed to parasite-expressed factors as well as enhanced cell senescence and that rigid RBCs contribute to disease pathology through sequestration of these cells and obstruction of the microvasculature. However, observation that rigidification is a feature common to clinical ant-malarial compounds tested here, suggest that its impact is primarily associated with clearance of infected RBCs by adsorption within the inter-endothelial clefts of the spleen. Finally, a small-scale screen of bisindole alkaloids revealed that reduced $\mathrm{RBC}$ deformability could serve as a predictor of anti-malarial activity in a compound. Together, these data, as well as recently published data [38], suggest that reduced cell deformability can serve as a rapid and sensitive biomarker for anti-malarial drug efficacy. The ability to assess cell deformability using massively parallelized microfluidic systems without the need for expensive reagents, make such systems ideal for largescale anti-malarial drug screens.

\section{Additional files}

Additional file 1: Figure S1. Biophysical response of uninfected red blood cells to chloroquine. Neither (A) chloroquine concentration nor (B) incubation time significantly affected the deformability of uninfected RBCs.

Additional file 2: Figure S2. Biophysical response of uninfected red blood cells to anti-malarial compounds. The anti-malarial compounds tested in this study did not significantly affect the deformability of uninfected RBCs.

Additional file 3: Figure S3. Biophysical response of uninfected red blood cells to bisindole alkaloid compounds. Bisindole alkaloids tested did not significantly affect the deformability of uninfected RBCs.

\section{Authors' contributions}

Experiments were conceived by HM, and SPD participated in experimental design and coordination. Multiplex Fluidic Plunger device was developed and validated by MEML. Molecular assays were performed by XD, ATS and KM. Chemical preparation and experimental resources were provided by YLD and KSR. Data analysis was performed by XD, KM and SPD. XD, KM, SPD, and
HM contributed to the preparation of the manuscript. All authors read and approved of the final manuscript.

\section{Author details}

${ }^{1}$ Department of Mechanical Engineering, University of British Columbia, 2054-6250 Applied Science Lane, Vancouver, BC V6T 1Z4, Canada. ${ }^{2}$ Department of Chemistry, University of British Columbia, Vancouver, BC, Canada.

${ }^{3}$ Department of Urologic Science, University of British Columbia, Vancouver, BC, Canada. ${ }^{4}$ Vancouver Prostate Centre, Vancouver General Hospital, Vancouver, BC, Canada.

\section{Acknowledgements}

The authors would like to thank Prof. Petra Rohrbach and Dr. Sarah Reiling, from the Institute of Parasitology at McGill University, for providing $P$. falciparum 3D7 parasites used in this study, as well as advice for in vitro culture techniques.

\section{Competing interests}

The authors declare that they have no competing interests.

Received: 19 August 2015 Accepted: 21 October 2015

Published online: 31 October 2015

\section{References}

1. WHO: World Malaria Report 2014. World Health Organization, Geneva. http://www.who.int/malaria/publications/world_malaria_report_2014/ en/. ISBN: 9789241564830

2. Black RE, Morris SS, Bryce J. Where and why are 10 million children dying every year? Lancet. 2003;361:2226-34

3. WHO: Global report on antimalarial efficacy and drug resistance. World Health Organization, Geneva. 2010. http://www.who.int/malaria/publications/atoz/9789241500470/en/. ISBN: 9789241500470.

4. Tangpukdee N, Duangdee C, Wilairatana P, Krudsood S. Malaria diagnosis: a brief review. Korean J Parasitol. 2009;47:93-102.

5. Flannery EL, Chatterjee AK, Winzeler EA. Antimalarial drug discovery approaches and progress towards new medicines. Nat Rev Microbiol. 2013;11:849-62.

6. Cranston HA, Boylan CW, Carroll GL, Sutera SP, Williamson JR, Gluzman IY, et al. Plasmodium-falciparum maturation abolishes physiologic red-cell deformability. Science. 1984;223:400-3.

7. Maier AG, Cooke BM, Cowman AF, Tilley L. Malaria parasite proteins that remodel the host erythrocyte. Nat Rev Microbiol. 2009;7:341-54.

8. Nash GB. EO: abnormalities in the mechanical properties of red blood cells caused by Plasmodium falciparum. Blood. 1989;74:855-61.

9. Hosseini SM, Feng JJ. How malaria parasites reduce the deformability of infected red blood cells. Biophys J. 2012;103:1-10.

10. del Portillo HA, Ferrer M, Brugat T, Martin-Jaular L, Langhorne J, Lacerda MVG. The role of the spleen in malaria. Cell Microbiol. 2012;14:343-55.

11. Nuchsongsin F, Chotivanich K, Charunwatthana P, Fausta O-S, Taramelli D, Day NP, White NJ, Dondorp AM. Effects of Malaria Heme Products on Red Blood Cell Deformability. Am J Trop Med Hyg. 2007;77:617-22.

12. Meshnick SR, Yang YZ, Lima V, Kuypers F, Kamchonwongpaisan S, Yuthavong Y. Iron-dependent free radical generation from the antimalarial agent artemisinin (qinghaosu). Antimicrob Agents Chemother. 1993:37:1108-14.

13. Huang S, Undisz A, Diez-Silva M, Bow H, Dao M, Han J. Dynamic deformability of Plasmodium falciparum-infected erythrocytes exposed to artesunate in vitro. Integr Biol Quant Biosci Nano Macro. 2013;5:414-22.

14. Huang S, Amaladoss A, Liu M, Chen H, Zhang R, Preiser P, et al. In vivo splenic clearance corresponds with in vitro deformability of red blood cells from Plasmodium yoelii infected mice. Infect Immun. 2014:82:2532-41.

15. Chotivanich K, Udomsangpetch R, Dondorp A, Williams T, Angus B, Simpson JA, Pukrittayakamee S, Looareesuwan S, Newbold Cl, White NJ. The mechanisms of parasite clearance after antimalarial treatment of Plasmodium falciparum malaria. J Infect Dis. 2000;182:629-33.

16. Kim CC, Wilson EB, DeRisi JL. Improved methods for magnetic purification of malaria parasites and haemozoin. Malar J. 2010;9:17. 
17. Mu J, Myers RA, Jiang H, Liu S, Ricklefs S, Waisberg M, et al. Plasmodium falciparum genome-wide scans for positive selection, recombination hot spots and resistance to antimalarial drugs. Nat Genet. 2010;42(3):268-71.

18. Vivas L, Rattray L, Stewart LB, Robinson BL, Fugmann B, Haynes RK, et al. Antimalarial efficacy and drug interactions of the novel semi-synthetic endoperoxide artemisone in vitro and in vivo. J Antimicrob Chemother. 2007;59(4):658-65.

19. Dahl EL, Rosenthal PJ. Multiple antibiotics exert delayed effects against the Plasmodium falciparum apicoplast. Antimicrob Agents Chemother. 2007;51(10):3485-90.

20. Jiménez-Díaz MB, Ebert D, Salinas Y, Pradhan A, Lehane AM, MyrandLapierre M-E, et al. (+)-SJ733, a clinical candidate for malaria that acts through ATP4 to induce rapid host-mediated clearance of Plasmodium. Proc Natl Acad Sci USA. 2014;111(50):E5455-62.

21. Myrand-Lapierre M-E, Deng X, Ang RR, Matthews K, Santoso AT, Ma H. Multiplexed fluidic plunger mechanism for the measurement of red blood cell deformability. Lab Chip. 2015;15:159-67.

22. Williams DE, Davies J, Patrick BO, Bottriell H, Tarling T, Roberge M, Andersen RJ. Cladoniamides A-G, tryptophan-derived alkaloids produced in culture by Streptomyces uncialis. Org Lett. 2008;10:3501-4.

23. Du Y-L, Ding T, Ryan KS. Biosynthetic O-methylation protects cladoniamides from self-destruction. Org Lett. 2013;15(10):2538-41.

24. Howard-Jones AR, Walsh CT. Staurosporine and rebeccamycin aglycones are assembled by the oxidative action of StaP, StaC, and RebC on chromopyrrolic acid. J Am Chem Soc. 2006;128:12289-98.

25. Chang F-Y, Kawashima SA, Brady SF. Mutations in the proteolipid subunits of the vacuolar $\mathrm{H}(+)$-ATPase provide resistance to indolotryptoline natural products. Biochemistry. 2014;53:7123-31.

26. Saliba KJ, Kirk K. pH regulation in the intracellular malaria parasite, Plasmodium falciparum. $\mathrm{H}(+)$ extrusion via a V-type $\mathrm{H}(+)$-ATPase. J Biol Chem. 1999;274:33213-9.

27. Friedman MJ. Oxidant damage mediates variant red cell resistance to malaria. Nature. 1979;280:245-7.
28. Loria P, Miller S, Foley M, Tilley L. Inhibition of the peroxidative degradation of haem as the basis of action of chloroquine and other quinoline antimalarials. Biochem J. 1999;339:363-70.

29. Brunet LR. Nitric oxide in parasitic infections. Int Immunopharmacol. 2001;1:1457-67.

30. Clark IA, Hunt NH. Evidence for reactive oxygen intermediates causing hemolysis and parasite death in malaria. Infect Immun. 1983;39:1-6.

31. Lee IS, Hufford CD. Metabolism of antimalarial sesquiterpene lactones. Pharmacol Ther. 1990;48(3):345-55.

32. Aweeka PFT, German PI. Clinical pharmacology of artemisinin-based combination therapies. Clin Pharmacokinet. 2008;47(2):91-102.

33. Navaratnam V, Mansor SM, Sit N-W, Grace J, Li Q, Olliaro DP. Pharmacokinetics of artemisinin-type compounds. Clin Pharmacokinet. 2000;39(4):255-70.

34. Eckstein-Ludwig U, Webb RJ, Van Goethem IDA, East JM, Lee AG, Kimura $\mathrm{M}$, et al. Artemisinins target the SERCA of Plasmodium falciparum. Nature. 2003:424(6951):957-61.

35. Spillman NJ, Allen RJW, Kirk K. Na + extrusion imposes an acid load on the intraerythrocytic malaria parasite. Mol Biochem Parasitol. 2013;189:1-4.

36. Spillman NJ, Allen RJW, McNamara CW, Yeung BKS, Winzeler EA, Diagana TT, et al. $\mathrm{Na}+$ regulation in the malaria parasite Plasmodium falciparum involves the cation ATPase PFATP4 and is a target of the spiroindolone antimalarials. Cell Host Microbe. 2013;13:227-37.

37. Fabre S, Prudhomme M, Rapp M. Protein kinase C inhibitors; structureactivity relationships in K252c-related compounds. Bioorg Med Chem. 1993;1:193-6.

38. Santoso AT, Deng X, Lee JH, Matthews K, Duffy SP, Islamzada E, McFaul SM, Myrand-Lapierre ME, Ma H. Microfluidic cell-phoresis enabling high-throughput analysis of red blood cell deformability and biophysical screening of antimalarial drugs. Lab Chip. 2015. doi:10.1039/C5LC00945F.

\section{Submit your next manuscript to BioMed Central and take full advantage of:}

- Convenient online submission

- Thorough peer review

- No space constraints or color figure charges

- Immediate publication on acceptance

- Inclusion in PubMed, CAS, Scopus and Google Scholar

- Research which is freely available for redistribution

Submit your manuscript at

www.biomedcentral.com/submit

C Biomed Central 